\title{
Head to head transcatheter heart valve comparisons: when theory becomes reality
}

\author{
Gabriela Tirado-Conte, German Armijo, Luis Nombela-Franco \\ Instituto Cardiovascular, Hospital Clínico San Carlos, IdISSC, Universidad Complutense, Madrid, Spain \\ Correspondence to: Luis Nombela-Franco, MD, PhD. Instituto Cardiovascular, Hospital Clínico San Carlos, IdISSC, Universidad Complutense, C/ \\ Prof. Martin Lagos s/n, 28040 Madrid, Spain. Email: luisnombela@yahoo.com. \\ Provenance: This is an invited Editorial commissioned by the Section Editor Yue Liu (The First Affiliated Hospital, Harbin Medical University, \\ Harbin, China). \\ Comment on: Feldman TE, Reardon MJ, Rajagopal V, et al. Effect of Mechanically Expanded vs Self-Expanding Transcatheter Aortic Valve \\ Replacement on Mortality and Major Adverse Clinical Events in High-Risk Patients With Aortic Stenosis: The REPRISE III Randomized Clinical \\ Trial. JAMA 2018;319:27-37.
}

Submitted May 19, 2018. Accepted for publication Jul 11, 2018.

doi: $10.21037 /$ cdt.2018.07.08

View this article at: http://dx.doi.org/10.21037/cdt.2018.07.08

Transcatheter aortic valve implantation (TAVI) is nowadays the standard treatment for severe aortic stenosis in symptomatic patients with extreme or high surgical risk (1-3) and a valid alternative to surgery in elderly patients at intermediate risk. This is the consequence of accumulating clinical experience, technological improvements and the promising results of several randomized clinical trials (4-6). Nevertheless, several limitations related to TAVI, such as conduction disturbances and paravalvular leak (PVL) remain an ongoing concern and an opportunity for improvement.

Mechanically expanded Lotus Valve system (MEV, Boston Scientific Corp) was designed to further minimize PVL rates and to insure a safe and controlled deployment. The MEV consists of 3 bovine pericardial tissue valve leaflets and a braided nitinol frame with an adaptive seal, which folds up on itself during valve expansion to fill the paravalvular space and diminish PVL. This device is mechanically expanded and capable to function early during the deployment process. The most interesting feature of this transcatheter heart valve (THV) is that it is capable of being fully retrievable and repositioned if necessary when the valve is already functioning in its final position. While limitation of balloon expandable THV is that the operator only has one chance to position it in the correct site, selfexpandable THV may be recaptured and repositioned until $80 \%$ of the deployment process. However, there is no possibility to retrieve the valve after it is fully released.
The REPRISE III (Safety and Efficacy Study of Lotus Valve for Transcatheter Aortic Valve Replacement) is a multicenter, randomized clinical trial that compared two competitive THV (7). Fifty-five participant centers from North America, Europe and Australia recruited 912 patients with severe symptomatic aortic stenosis and high or extreme surgical risk, which were randomized 2:1 to receive either MEV ( $\mathrm{n}=607)$ or the self-expanding CoreValve (SEV, CoreValve Classic or Evolut-R; Medtronic) ( $\mathrm{n}=305)$. Feldman et al. reported 1-year results with particular emphasis in safety and effectiveness in terms of mortality, stroke, bleeding and vascular complications, acute kidney injury and PVL. Authors and supporters should be commended for performing a direct head-to-head comparison between two THVs, which is the best scientific approach to obtain definitive conclusions. Until now, there was only a direct randomized trial comparing two available THVs (8), although several publications indirectly compared some THVs (9-11). With the expansion of the technique to intermediate and lower risk patients and several THVs available in the market, studies with direct comparisons will be necessary in order to define the best treatment and THV choice for each patient.

Major findings of the REPRISE III were that both systems had similar rates of the primary safety end-point (20.3\% in the MEV group vs. $17.2 \%$ in SEV group, $\mathrm{P}=0.003$ for non-inferiority), with some differences in secondary 
end-points. While, thrombosis, permanent pacemaker (PPM) implantation and valve hemodynamics favored SEV, rate of significant PVL, valve malpositioning and need for a second valve was less frequent in MEV group. The grade of PVL is linearly related to increased mortality, especially in cases with moderate-to-severe PVL (4). Thus, residual PVL has been the major limitation of TAVI compared to surgery. Technological improvements in prosthesis design as well as anatomical considerations (multimodality imaging, optimized valve sizing) and deployment technique have contributed to lowered post-TAVI PVL severity with newer generation valves (12). A major limitation of the trial is that most patients in the SEV group were treated with the old-generation of the CoreValve system. Residual PVL has been traditionally greater with SEV, especially with previous generation, compared to balloon expandable THV and to newer generation of $\operatorname{SEV}(9,13,14)$. In fact, in the REPRISE III, a post hoc analysis with limited number of patients treated with the Evolut-R showed that PVL was below $<3 \%$ without differences compared to Lotus valve $(<1 \%)$. This data suggests that Lotus valve reached surgical rates of significant PVL and other THV are in the right direction with almost similar results (7). One may wonder, if this difference in the rate of PVL would remain in a complete comparison versus Evolut-R or Sapien-3.

While several efforts have been made to reduce PVL, conduction disturbances after TAVI have not been given the same attentiveness and their clinical impact may be minimized (15). The impact of conduction disturbances at short-term are increased hospital stay and costs $(16,17)$. Furthermore, left bundle branch block (LBBB) post-TAVI has been associated with higher mortality (18), a trend toward increased hospitalization, a lack of left ventricular ejection fraction (LVEF) recovery, poorer functional class at follow-up, and increased permanent pacemaker (PPM) placement (19). Also, PPM implantation is not innocuous. Right ventricular pacing has been shown to cause reduction in LVEF and cardiac output due to interventricular dyssynchrony (20). Although, some studies revealed similar mid-term mortality in patients with PPM (21), other studies showed higher mortality and heart failure admission rates (22). In addition, recent studies showed poorer outcomes in patients with pacemaker after TAVI at longer follow-up (16). Unfortunately, the rates of PPM implantation have even increased with newer generation THV $(15,23)$ and definitively, this would have an impact when expanding the indications to younger patients. In this study, patients in the Lotus arm had a PPM implantation rate of $35.5 \%$ and $41.4 \%$ at 30 -day and 1 year, respectively. Previous studies have showed similar results in patients receiving Lotus Valve system, revealing baseline conduction abnormalities, deeper implantation and annular oversizing as predictors of PPM implantation (24). In this trial, depth of implant was similar between MEV and SEV. Unfortunately, data about baseline EKG or prosthesis oversizing was not reported, as well as the incidence of new LBBB. Meanwhile, in other non-randomized studies LBBB exceeded $50 \%$ in patients receiving MEV (24). A rate of more than $70 \%$ of the patients developing a new conduction disturbance (either pacemaker or de novo LBBB) is excessively high and make this TVH difficult to justify when other THV types had a pacemaker rate around $5 \%$ to $10 \%(25,26)$. Therefore, the benefits and advantages of a fully repositionable THV with a very low aortic regurgitation (AR) rate may be jeopardized by the excessive radial force and damage caused in the conduction system. Second generation MEV: the Lotus Edge valve system device may address this issue with the new Depth Guard $^{\mathrm{TM}}$ technology that limit left ventricular outflow tract (LVOT) interaction, which theoretically might reduce conduction abnormalities. Additional design improvements are a flexible delivery system, lower profile access $(14 \mathrm{~F} / 15 \mathrm{~F})$ and more valve sizes (21 and $29 \mathrm{~mm}$ ). However, first and second-generations MEV are currently off the market. The company released a voluntary recall due to issues with the locking mechanism. The problem stems from premature release of a pin connecting the valve to the delivery system, which is consequence of a manufacturing error that leads to additional pressure in the pin system. Boston scientific has announced the return of Lotus Edge THV but it has been delayed several times, first in November 2017 and February 2018 , lastly until 2019 , apparently since the company is pending final testing and regulatory approval timelines. Nevertheless, one might speculate if the company could be considering focusing its efforts in other THV technology rather than returning to MEV.

In summary, the more outstanding feature of the REPRISE III is that it is one of the first international headto-head randomized clinical trial that compare two THVs, demonstrating that MEV and SEV are comparable in terms of the primary safety and effectiveness end-points. PVL and PPM implantation continue to be a drawback for TAVI and may be inversely related. Lotus Valve system achieved the lower PVL rate, probably by increasing the strength to seal the paravalvular space. However, it resulted in larger degree of LVOT compression causing higher conduction 
abnormalities. MEV may be particularly useful in patients with severe, asymmetric aortic valve calcification or with high eccentricity annulus index, as in bicuspid aortic valve, with the intention of minimizing malpositioning and PVL. However, a high rate of conduction disturbances should be expected. Thus, future studies will have to determine which valve is the best valve for each specific group of patients.

\section{Acknowledgements}

None.

\section{Footnote}

Conflicts of Interest: L Nombela-Franco has served as a proctor for Abbott and has received speaker honoraria from Edwards Lifesciences Inc. Other authors have no conflicts of interest to declare.

\section{References}

1. Leon MB, Smith CR, Mack M, et al. Transcatheter aorticvalve implantation for aortic stenosis in patients who cannot undergo surgery. N Engl J Med 2010;363:1597-607.

2. Kodali SK, Williams MR, Smith CR, et al. Two-Year Outcomes after Transcatheter or Surgical Aortic-Valve Replacement. N Engl J Med 2012;366:1686-95.

3. Adams DH, Popma JJ, Reardon MJ, et al. U.S. CoreValve Clinical Investigators. Transcatheter aortic-valve replacement with a self-expanding prosthesis. $\mathrm{N}$ Engl J Med 2014;370:1790-8.

4. Leon MB, Smith CR, Mack MJ, et al. Transcatheter or Surgical Aortic-Valve Replacement in Intermediate-Risk Patients. N Engl J Med 2016;374:1609-20.

5. Søndergaard L, Steinbrüchel DA, Ihlemann N, et al. TwoYear Outcomes in Patients With Severe Aortic Valve Stenosis Randomized to Transcatheter Versus Surgical Aortic Valve Replacement: The All-Comers Nordic Aortic Valve Intervention Randomized Clinical Trial. Circ Cardiovasc Interv 2016;9.

6. Reardon MJ, Van Mieghem NM, Popma JJ, et al. Surgical or Transcatheter Aortic-Valve Replacement in IntermediateRisk Patients. N Engl J Med 2017;376:1321-31.

7. Feldman TE, Reardon MJ, Rajagopal V, et al. Effect of Mechanically Expanded vs Self-Expanding Transcatheter Aortic Valve Replacement on Mortality and Major Adverse Clinical Events in High-Risk Patients With Aortic Stenosis: The REPRISE III Randomized Clinical Trial.
JAMA 2018;319:27-37.

8. Abdel-Wahab M, Mehilli J, Frerker C, et al. Comparison of Balloon-Expandable vs Self-expandable Valves in Patients Undergoing Transcatheter Aortic Valve Replacement: the CHOICE Randomized Clinical Trial. JAMA 2014;311:1503-14.

9. Soliman OII, El Faquir N, Ren B, et al. Comparison of valve performance of the mechanically expanding Lotus and the balloon-expanded SAPIEN3 transcatheter heart valves: an observational study with independent core laboratory analysis. Eur Heart J Cardiovasc Imaging 2018;19:157-67.

10. Chieffo A, Buchanan GL, Van Mieghem NM, et al. Transcatheter aortic valve implantation with the Edwards SAPIEN versus the Medtronic CoreValve Revalving system devices: a multicenter collaborative study: the PRAGMATIC Plus Initiative (Pooled-RotterdAmMilano-Toulouse In Collaboration). J Am Coll Cardiol 2013;61:830-6.

11. Wöhrle J, Gonska B, Rodewald C, et al. Transfemoral aortic valve implantation with the repositionable Lotus valve compared with the balloon-expandable Edwards Sapien 3 valve. Int J Cardiol 2015;195:171-5.

12. Binder RK, Webb JG, Willson AB, et al. The impact of integration of a multidetector computed tomography annulus area sizing algorithm on outcomes of transcatheter aortic valve replacement: a prospective, multicenter, controlled trial. J Am Coll Cardiol 2013;62:431-8.

13. Nombela-Franco L, Ruel M, Radhakrishnan S, et al. Comparison of hemodynamic performance of selfexpandable CoreValve versus balloon-expandable Edwards SAPIEN aortic valves inserted by catheter for aortic stenosis. Am J Cardiol 2013;111:1026-33.

14. Del Trigo M, Dahou A, Webb JG, et al. Self-expanding Portico Valve Versus Balloon-expandable SAPIEN XT Valve in Patients With Small Aortic Annuli: Comparison of Hemodynamic Performance. Rev Esp Cardiol (Engl Ed) 2016;69:501-8.

15. Auffret V, Puri R, Urena M, et al. Conduction Disturbances After Transcatheter Aortic Valve Replacement: Current Status and Future Perspectives. Circulation 2017;136:1049-69.

16. Fadahunsi OO, Olowoyeye A, Ukaigwe A, et al. Incidence, Predictors, and Outcomes of Permanent Pacemaker Implantation Following Transcatheter Aortic Valve Replacement: Analysis From the U.S. Society of Thoracic Surgeons/American College of Cardiology TVT Registry. JACC Cardiovasc Interv 2016;9:2189-99. 
17. Chevreul K, Brunn M, Cadier B, et al. Cost of transcatheter aortic valve implantation and factors associated with higher hospital stay cost in patients of the FRANCE (FRench Aortic National CoreValve and Edwards) registry. Arch Cardiovasc Dis 2013;106:209-19.

18. Houthuizen P, Van Garsse LA, Poels TT, et al. Left bundle-branch block induced by transcatheter aortic valve implantation increases risk of death. Circulation 2012;126:720-8.

19. Urena M, Webb JG, Cheema A, et al. Impact of newonset persistent left bundle branch block on late clinical outcomes in patients undergoing transcatheter aortic valve implantation with a balloon-expandable valve. JACC Cardiovasc Interv 2014;7:128-36.

20. Dizon JM, Nazif TM, Hess PL, et al. Chronic pacing and adverse outcomes after transcatheter aortic valve implantation. Heart 2015;101:1665-71.

21. Urena M, Webb JG, Tamburino C, et al. Permanent pacemaker implantation after transcatheter aortic valve implantation: impact on late clinical outcomes and left ventricular function. Circulation 2014;129:1233-43.

22. Nazif TM, Dizon JM, Hahn RT, et al. Predictors and

Cite this article as: Tirado-Conte G, Armijo G, NombelaFranco L. Head to head transcatheter heart valve comparisons: when theory becomes reality. Cardiovasc Diagn Ther 2018;8(4):552-555. doi: 10.21037/cdt.2018.07.08
Clinical Outcomes of Permanent Pacemaker Implantation After Transcatheter Aortic Valve Replacement: The PARTNER (Placement of AoRtic TraNscathetER Valves) Trial and Registry. JACC Cardiovasc Interv 2015;8:60-9.

23. De Torres-Alba F, Kaleschke G, Diller GP, et al. Changes in the Pacemaker Rate After Transition From Edwards SAPIEN XT to SAPIEN 3 Transcatheter Aortic Valve Implantation: The Critical Role of Valve Implantation Height. JACC Cardiovasc Interv 2016;9:805-13.

24. Rampat R, Khawaja MZ, Hilling-Smith R, et al. Conduction abnormalities and permanent pacemaker implantation after transcatheter aortic valve replacement using the repositionable LOTUS device: the United Kingdom experience. JACC Cardiovasc Interv 2017;10:1247-53.

25. Reichenspurner H, Schaefer A, Schäfer U, et al. SelfExpanding Transcatheter Aortic Valve System for Symptomatic High-Risk Patients With Severe Aortic Stenosis. J Am Coll Cardiol 2017;70:3127-36.

26. Nombela-Franco L. New Valves May Overcome Weaknesses of Transcatheter Aortic Valve Replacement. J Am Coll Cardiol 2017;70:3137-9. 